\title{
Impact of a Collaborative Clinical Trials Methodology Course on Trainees' Funding and Clinical Trials Activity
}

\author{
Shirley Yang and William J. Meurer, MD, MS
}

University of Michigan Medical School, Ann Arbor, MI, United States

National Institute of

Neurological Disorders and Stroke

\section{Background}

- Clinical trials are challenging, and many junior investigators lack the skills to move through the phases in a coherent way

- Achieving project funding is also competitive, with a U.S. National Institutes of Health (NIH) funding success rate of $20.2 \%$ in $2018^{1}$

- The U.S. National Institute of Neurological Disorders and Stroke Clinical Trials Methodology Course (CTMC) was created to provide relevant training for junior investigators to promote well-designed clinical trials

- The course relies on cross university collaborations to present webinars, in-person conferences, and small group experiences (see Project and Contact Information)

- This study aims to explore the impact of CTMC by determining the trajectory of NIH funding and clinical trials activity in trainees after completion of the course

\section{Methods}

- The online databases NIH RePORTER and ClinicalTrials.gov were searched respectively to determine NIH funding and clinical trials activity of each CTMC trainee between 2014-2017

- Data was collected on the Project Start Date of the first NIH grant and the Study Start Date of the first non-withdrawn clinical trial for each trainee after taking CTMC (as of early 2019)

- The time to receiving NIH grant or being involved in clinical trial was calculated as the difference between the Project/Study Start Date and the trainee's CTMC date

- The CTMC date of a trainee was designated as January $1^{\text {st }}$ of the year following course completion

- $\mathrm{NIH}$ grants and clinical trials prior to taking CTMC were noted

\section{Results}

Total Funding and Clinical Trial Activity of CTMC Trainees 2014-2017 ( $n=131)$

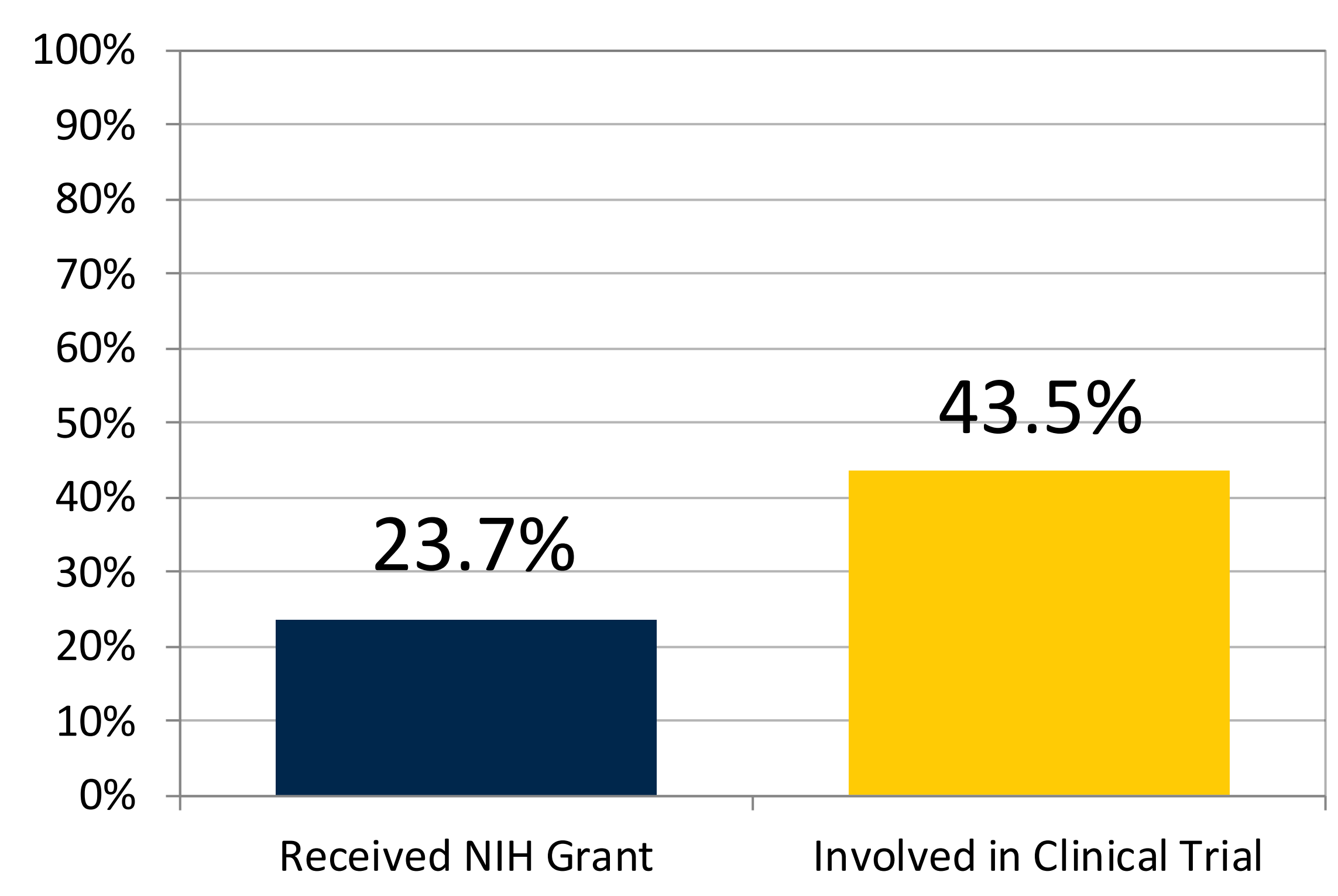

Figure 1. Of the 131 CTMC trainees between 2014-2017, 23.7\% received a NIH grant and 43.5\% became involved in a clinical trial after taking the course

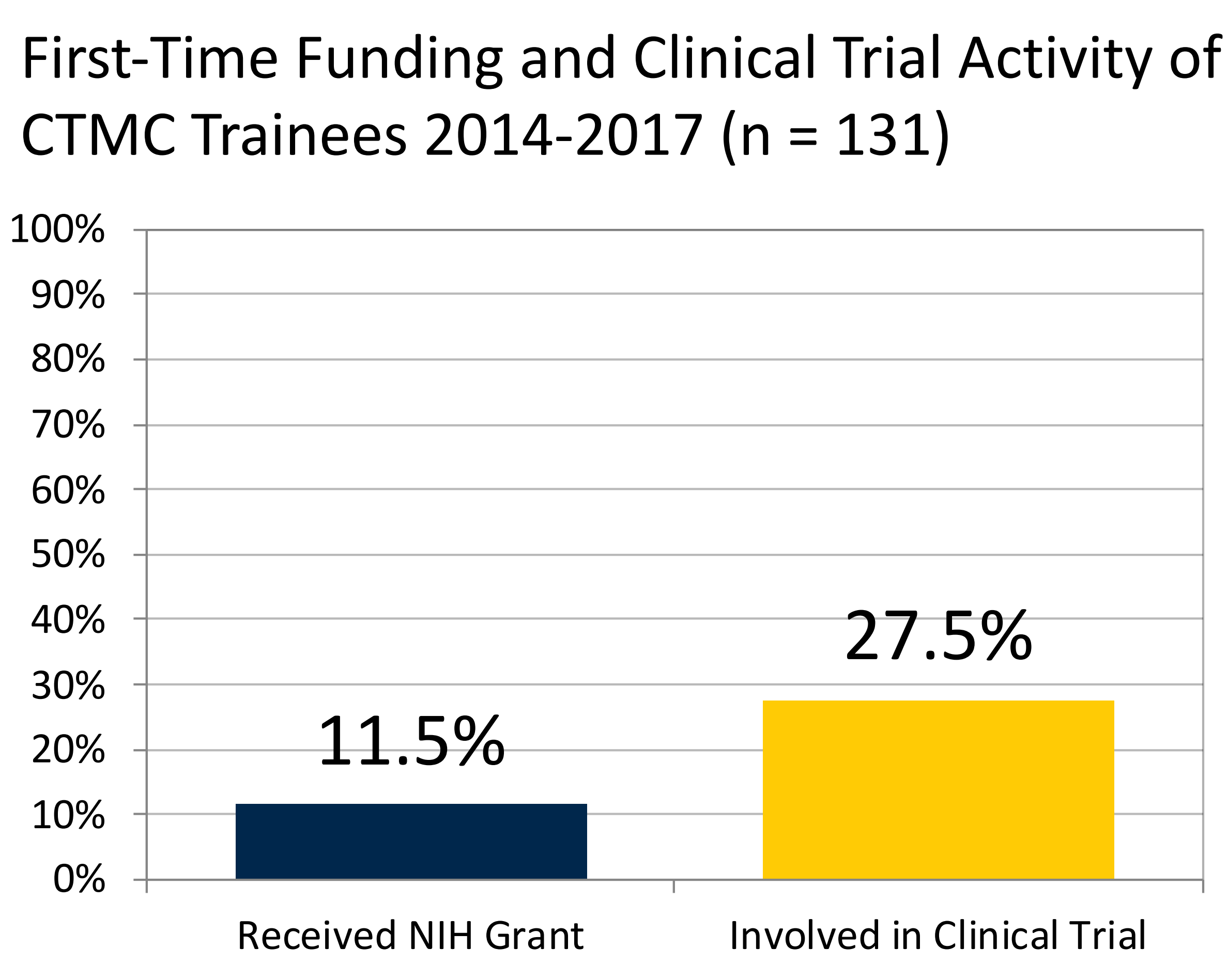

Figure 2. Excluding those with prior experience, $11.5 \%$ of all trainees received their first-ever $\mathrm{NIH}$ grant and $27.5 \%$ became involved in their firstever clinical trial following completion of CTMC
Median Time to Achieving Funding and Clinical Trial Activity of CTMC Trainees 2014-2017 ( $n=131$ )

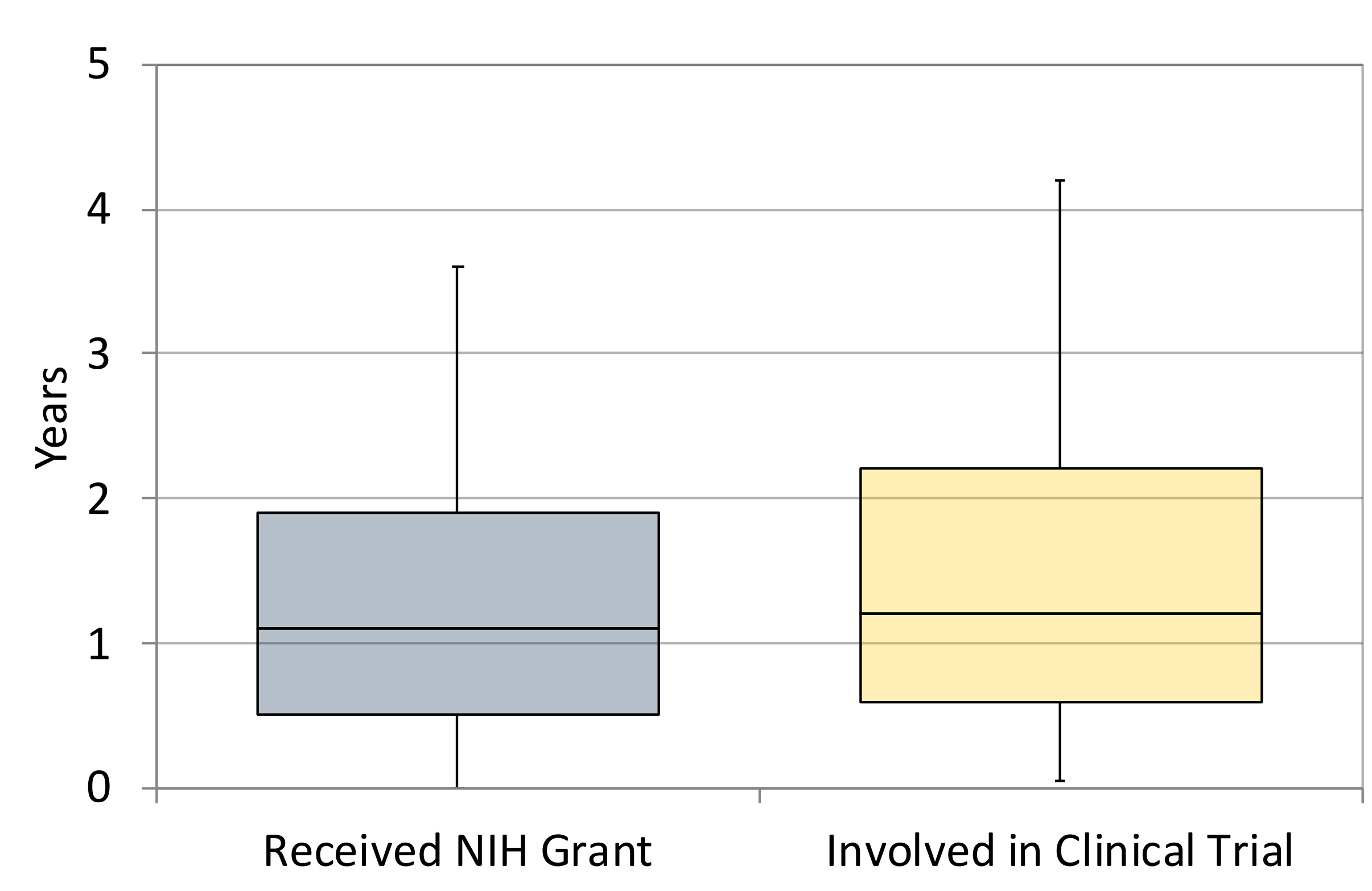

Figure 3. The median time to achieving a $\mathrm{NIH}$ grant was 1.1 years (IQR 0.5-1.9 years) and the median time to becoming involved in a clinical trial was 1.2 years (IQR 0.6-2.2 years) after completion of CTMC
Yearly Trajectory of Total Funding and Clinical Trial Activity of CTMC Trainees

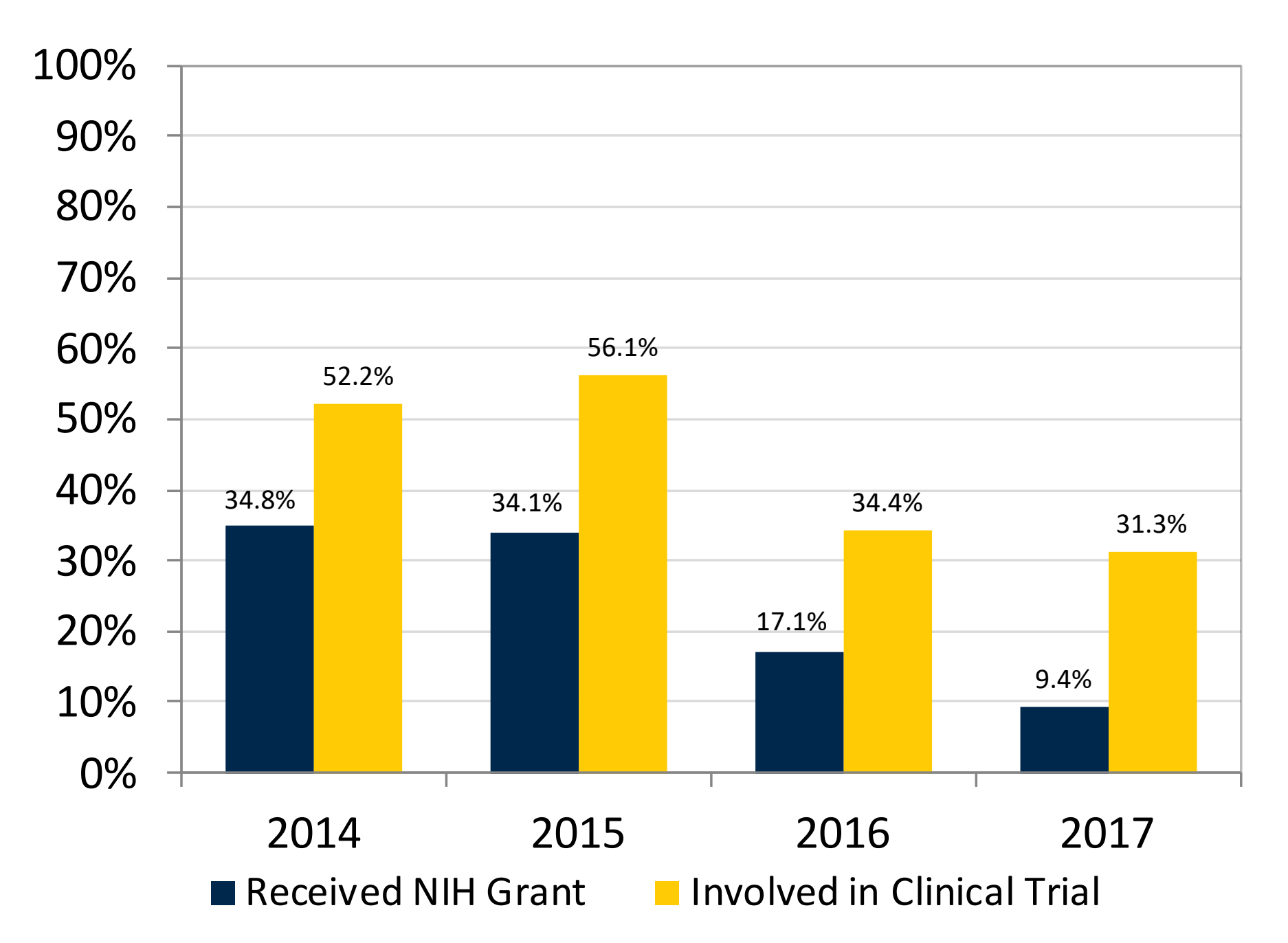

Figure 4. The percentage of trainees with $\mathrm{NIH}$ funding and clinical trial activity increases with time after course completion

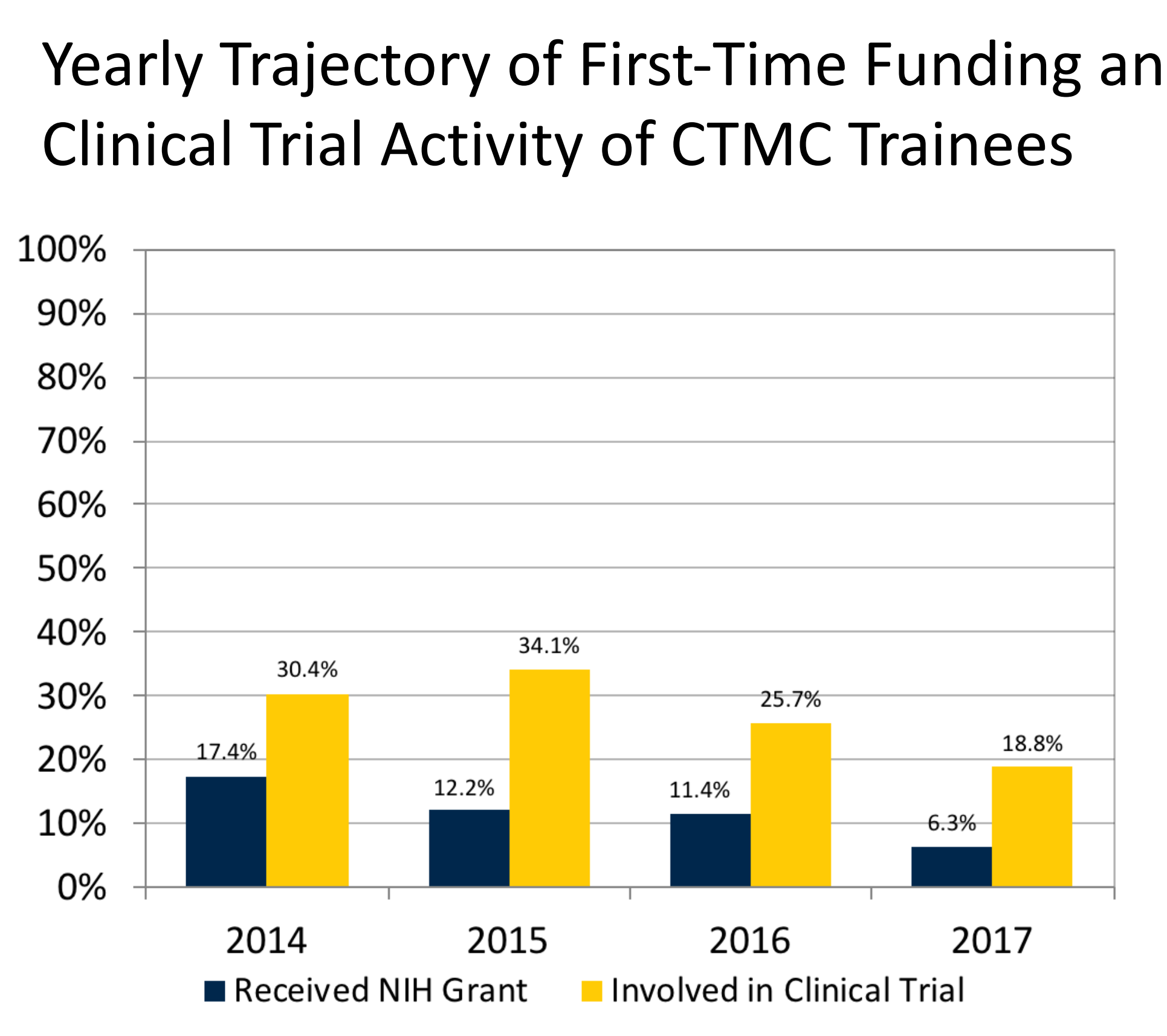

Figure 5. The percentage of trainees with firstever NIH funding and clinical trial activity also increases with time after course completion

\section{Conclusions}

- These results suggest that completing CTMC may help promote successful funding and clinical trials by both experienced and nonexperienced junior investigators

- With more time, an increasing number of CTMC trainees may be able to secure funding and have clinical trials activity

- Limitations of this study include not having a control group for comparison and not accounting for other sources of funding and clinical trial activity

- Future directions include contacting investigators and cross-referencing their self-reported achievements to provide a detailed examination of the impact of CTMC on their research careers

Yearly Trajectory of Median Time to Achieving NIH Funding of CTMC Trainees

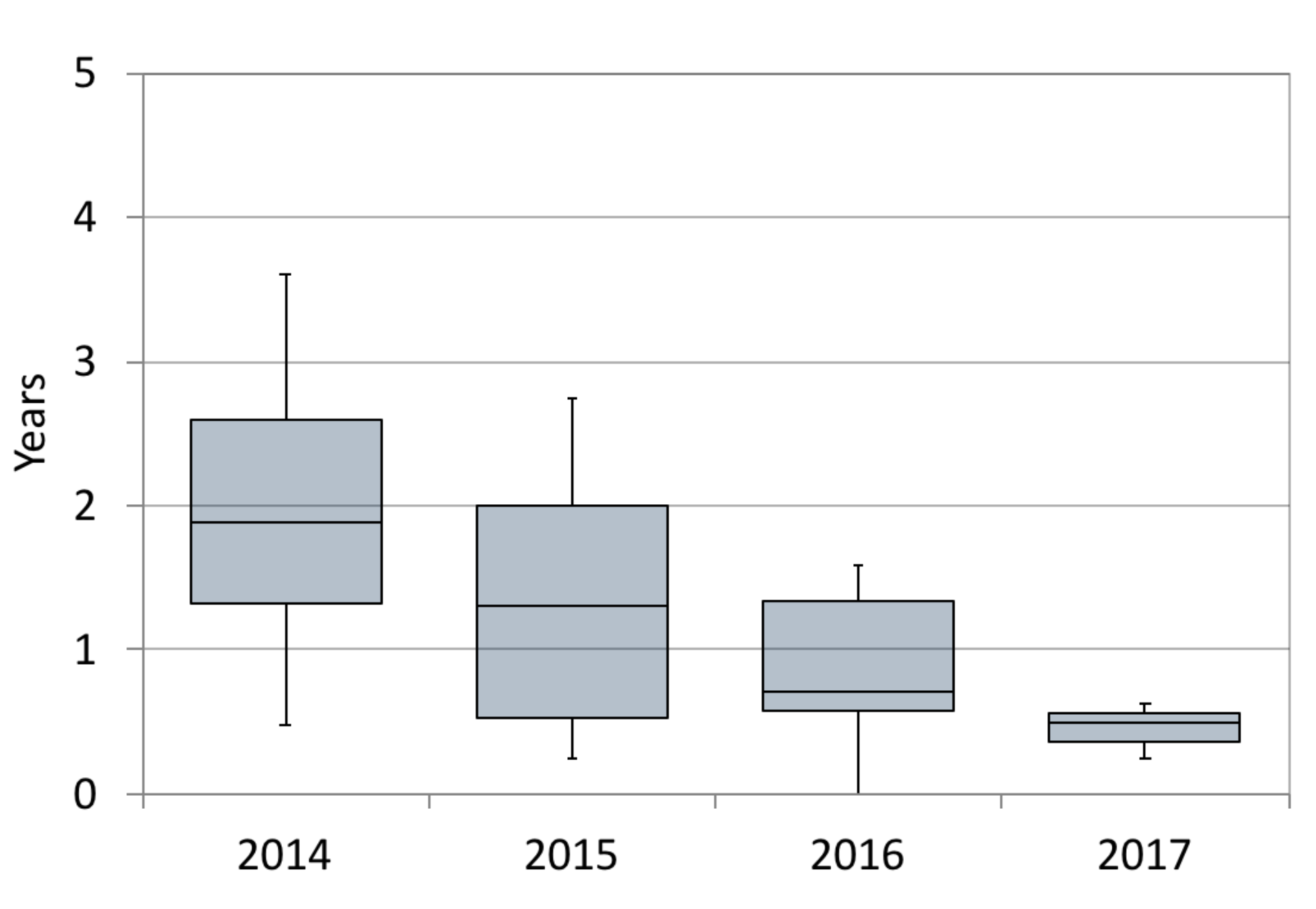

Figure 6. The median time to achieving NIH funding increases with time after course completion
Yearly Trajectory of Median Time to Clinical Trial Activity of CTMC Trainees

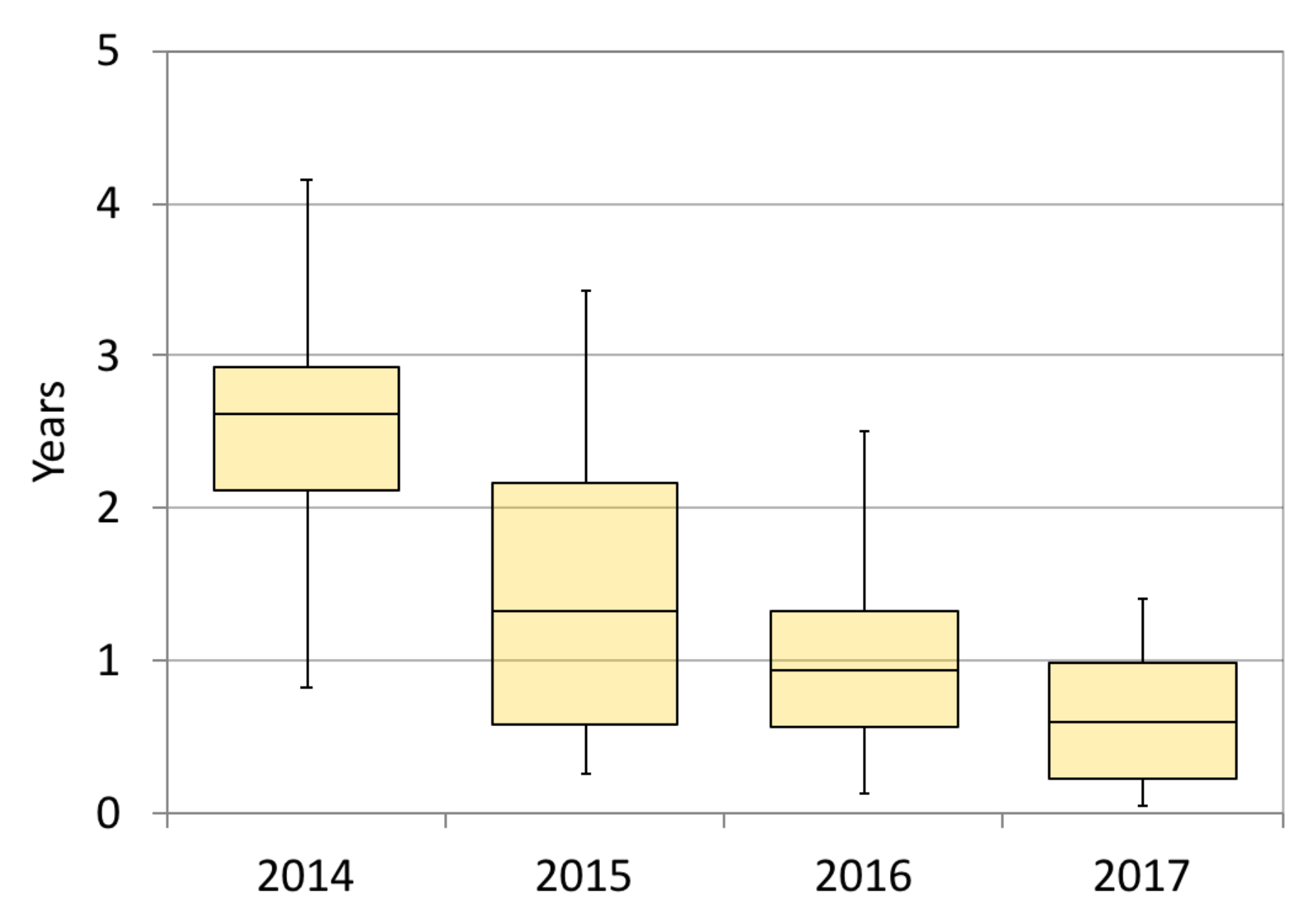

Figure 7. The median time to achieving clinical trial activity also increases with time after course completion

\section{Project and Contact Information}

- CTMC is a project based, immersive course. Our materials can be found at http://neurotrials.training (scan QR code)

- Contact information: Dr. William Meurer, wmeurer@umich.edu and Twitter@willmeurer

\section{Acknowledgements and References}

- The CTMC course is supported by the NIH grant \#R25 NS088248

- References:

1. National Institute of Health. Research Project Success Rate by NIH Institute for 2018. (https://report.nih.gov/success rates/Success BylC.cfm) 